\title{
The 10-year trend in drug prescriptions for attention-deficit/hyperactivity disorder (ADHD) in Germany
}

\author{
Thomas Grimmsmann ${ }^{1}\left(\mathbb{D} \cdot\right.$ Wolfgang Himmel $^{2}($ )
}

Received: 13 February 2020 / Accepted: 26 June 2020 / Published online: 17 August 2020

(C) The Author(s) 2020

\begin{abstract}
Purpose The aim of this study was to analyse whether the global trend in drug prescriptions for attention-deficit hyperactivity disorders (ADHD), as observed during the last years and often criticized as medicalization, have remained stable or shifted. Methods This observational study was based on a secondary analysis of data from a large German database including patients with an ADHD diagnosis between 2008 and 2018. Prescription data comprised all important ADHD drugs.

Results A total of 620 practices delivered data from a total of 77,504 patients ( $31 \%$ of them females) with a diagnosis of AHDH. Nearly 38\% $(29,396 / 77,504)$ of all patients received, at least, one prescription for an ADHS medicine between 2008 and 2018. The number of patients receiving a drug steadily increased annually until 2012 and then slowly fell, but unevenly distributed across the age groups. While the number of younger patients $(\leq 16$ years) receiving a prescription fell by $24 \%$ and the defined daily doses (DDDs) remained stable, the number of patients between 17 and 24 years receiving a prescription increased by $113 \%$ and the DDDs by $150 \%$. Respectively, the number of older adults ( $\geq 25$ years) with a prescription increased by $355 \%$ and the DDDs by $515 \%$. Nearly onethird of older adults received an ADHD medicine only once.

Conclusion The ever-increasing prescription of ADHD medicines stopped some years ago for children. ADHS and its pharmacological management are increasingly observed among older adolescents and adults, with a different pattern of drug persistence compared with children.
\end{abstract}

Keywords Attention-deficitdisorder with hyperactivity $\cdot$ Drug prescriptions $\cdot$ Cohortstudies $\cdot$ Ambulatory care $\cdot$ Methylphenidate

\section{Introduction}

Attention-deficit/hyperactivity disorder (ADHD) is among the most common neurobehavioural problem afflicting children and adolescents. Individuals suffering from this

Electronic supplementary material The online version of this article (https://doi.org/10.1007/s00228-020-02948-3) contains supplementary material, which is available to authorized users.

Thomas Grimmsmann

t.grimmsmann@mdk-mv.de

Wolfgang Himmel

whimmel@gwdg.de

1 German Health Insurance Medical Service (MDK) Mecklenburg-Vorpommern, 19059 Schwerin, Germany

2 Department of General Practice, University of Göttingen, Humboldtallee 38, 37073 Goettingen, Germany disorder exhibit hyperactivity, inattention, impulsivity and problems in social interaction and academic performance [1]. In a substantial number of cases, the disorder does not remit in puberty but persists into adulthood [2]. Whether late-onset ADHD exists is controversially discussed [3, 4]. The estimated overall population prevalence of ADHD is $7.2 \%$ (95\% confidence interval: 6.7 to 7.8) [5]; the administrative prevalence of ADHD is $4.33 \%$ (95\% CI: $4.31-4.34 \%$ ) for children and adolescents in Germany; for adults, the prevalence is far lower $[6,7]$.

The number of prescriptions and the number of children who take ADHD drugs have increased dramatically in various European countries [8], and recently, the same trend has been observed in adults as well $[7,9]$. If we consider the overall drug prevalence in Germany, there was an increase from $1.3 \%$ of children between 5 and 9 years or $2.9 \%$ of children between 10 and 14 years in 2005 up to $2.0 \%$ or $4.4 \%$, respectively, in the following years [8]. This upward trend seems to have reached a 
plateau or has been slightly decreasing in recent years for children, while for adults, it is still increasing [7, 10].

There are several concerns about the pharmacological management of ADHD. Methylphenidate, the most commonly prescribed medicine, is associated with an increased risk of non-serious adverse events, such as sleep problems and decreased appetite [11]. Moreover, the widespread use of ADHD medicines, especially the use of methylphenidate in children, has long been criticized as medicalization [12], i.e., the definition and treatment of typical human experiences and emotions as medical conditions under the authority of physicians.

Despite these concerns, new guidelines on the management of ADHD recommend a more liberal use of these drugs. For example, the guideline of the National Institute for Health and Care Excellence (NICE) recommends pharmacotherapy as first-line treatment for adults [13], although research of pharmacotherapy for adults is still largely lacking [2], and methylphenidate is the first-line pharmacological treatment for children over five and adolescents if symptoms are still causing a persistent significant impairment in at least one domain after environmental modifications have been implemented and reviewed. In other words, NICE focuses on the presence of significant impairment in the different domains of everyday life, rather than using the previously used terms of mild, moderate and severe ADHD [13]. Similarly, an updated German guideline (AWMF) [14] recommends these medicines not only for severe cases, as in former versions, but also for moderate cases. These updates were criticized by the public [15] as well as scientists [16] due to a supposed further increase in drug prescriptions. To date, however, we do not know whether the new guidelines led, indeed, to increased drug prescriptions.

Additionally, and in remarkable contrast to these concerns, we witness a constantly repeated warning of an allegedly insufficient awareness of "adult ADHD" [17] and its possible undertreatment $[7,18,19]$, accompanied by an increase of administrative prevalence of adult ADHD and a new clinical picture, coined late-onset ADHD [6].

In other words, we face a mixture of fears of overtreatment of children/adolescents and undertreatment of adults. In this situation, new and valid data that highlight the prescription of these drugs are key for optimal treatment and rational discourse. According to Beau-Lejdstrom and Magno Zito [20], it is essential to expand our knowledge of medication use patterns from large observational studies, for example, by measuring the prevalence of ADHD medication use across the age groups and in terms of duration (i.e. persistence of ADHD medication use). The aim of this study was to analyse whether the global trends in ADHD prescriptions observed during the last years have remained stable or shifted, with a special focus on and comparison between children, adolescents and adults.

\section{Material and methods}

\section{Design}

This observational study was based on a secondary analysis of data from a large database provided by IMS/IQVIA [21]. For data preparation, analysis and reporting of the results, we used the RECORD checklist for reporting observational studies [18].

\section{Database}

The IMS ${ }^{\circledR}$ Disease Analyzer contains anonymized data obtained from the practice computers from office-based physicians specializing in various disciplines. The database records, in addition to other data, ICD-10-coded diagnoses, prescriptions, referrals and dates of appointments. The database contains data from 2498 practices [21] and appears to be representative of prescriptions issued by statutory health insurance (SHI)-accredited physicians [22].

The authors obtained data from a part of the database that included all patients with at least one diagnosis of ADHD (ICD-10-CM: F90 attention-deficit hyperactivity disorders) between 2008 and 2018, including information on physician specialty, patient's age and sex, diagnosis expressed as ICD 10 codes (up to level 4), date of diagnosis and referrals. Prescription data comprised date of visit, product expressed as an anatomical therapeutic chemical (ATC) code, strength and pack size.

All data are physician-related and patient-related only for a single practice so that a patient could not be followed if he or she changed practice.

\section{Definitions and analysis}

ADHD drugs Analysis included drugs approved for treatment of ADHD in Germany: dexamfetamine, lisdexamfetamine and methylphenidate as stimulants and atomoxetine and guanfacine as non-stimulant drugs.

Measurement of prescriptions and persistence Defined daily doses (DDDs) were calculated from the prescribed strength and pack size for each prescription. For persistence, according to the date of the first prescription, we calculated the months between the first and last prescription until there were no further prescriptions within 360 days.

Age groups For some analyses, we divided the patients into 3 age groups as follows: children, adolescents (16 years and younger), young persons (from 17 years to 24 years) and adults (25 years and older), similar to a classification in a French observational cohort study that assessed patterns of methylphenidate use in children and adults [23]. We 
deliberately deviated from the usual classification of children, adolescents and adults since - compared with our knowledge on children and young adolescents-our knowledge on ADHD medication in the critical phase of development and young adulthood is limited.

Statistical analysis In most instances, the unit of analysis was the ADHD diagnosed patient with a prescribed ADHD medication. Only descriptive statistics, such as absolute and relative frequencies, as well as the means, medians and interquartile ratios (IQRs), were calculated. For a sensitivity analysis, we changed the stop criterion for persistence from 360 days to a gap of up to 180 days.

\section{Results}

\section{Patients and prescriptions}

A total of 620 practices, including 437 primary care physicians, 92 paediatricians, 64 neurologists and 27 psychiatrists, delivered data from a total of 77,504 patients with a diagnosis of ADHD. The patients were, on average, 18.9 (median: 12) years old at the time of the first diagnosis of ADHD and/or the first ADHD prescription; $30.8 \%$ of them were female.

Nearly $38 \%(29,396 / 77,504)$ of all patients received at least one prescription for an ADHD medicine during the time period under study; $26 \%(7704 / 29,396)$ of them were female.

The number of patients receiving a drug steadily increased annually from 6613 in 2008 to 8969 in 2012 and then fell to 7533 in 2018 (Fig. 1), with a highly unequal distribution between children/adolescents on the one side and adults on the other side. The number of younger persons ( $\leq 16$ years) receiving ADHD medicine continuously fell from 6767 in 2011 to 4085 in 2018, and the number of older persons ( $\geq 17$ years) continuously rose throughout the ten years under study (Fig. 1). In those $\geq 17$ and $\leq$ 24 years, there was an increase of more than $100 \%$ (from 839 persons in 2008 to 1791 in 2018); in those $\geq 25$ years, there was an increase of more than $350 \%$ (from 364 persons in 2008 to 1657 in 2018). While these two groups together represented $18 \%$ of all persons receiving ADHD medicine in 2008, they represented $46 \%$ in 2018.

The situation is similar if we look at the quantity of medicines. The quantity rose from $1,037,210$ DDDs in 2008 to $1,537,449$ DDDs in 2018, an increase of $48 \%$, while the number of patients increased by only $14 \%$ (Fig. 2). Consequently, the increase in the number of DDDs was due to a continuous increase in the average number of DDDs per patient from 157 DDDs/patient/year in 2008 to 204 DDDs in 2018, with a somewhat stronger increase in older adults than in the remainder of the studied population (see Table; Appendix A). Like the number of patients, the total number of DDDs for younger persons decreased after 2012 but only slightly and remained stable for the following years. For older adults ( $\geq 25$ years), we saw an increase of more than $500 \%$ in the number of DDDs, from 57,029 in 2008 to 350,656 in 2018 (Fig. 2), while the number for those $\geq 17$ and $\leq 24$ years more than doubled (from 141,975 to 355,486 ).

Methylphenidate (Ritalin) is still by far the most prescribed medicine for ADHD, followed by lisdexamfetamine, which was first prescribed in 2013, and atomoxetine. The three substances differ in regard to the targeted population. Methylphenidate was the dominant substance 10 years ago, with more than $90 \%$ of patients receiving this drug. This number decreased for children/adolescents from $92 \%$ to $81 \%$ of the patients (or, in terms of DDD, from $93 \%$ to $70 \%$ ) and from $98 \%$ to $91 \%$ for adults (from $99 \%$ to $91 \%$ DDDs). Lisdexamfetamine increased, especially in children/ adolescents (2018: 21\% patients; 23\% DDD). The share of
Fig. 1 Patients with an ADHD prescription; according to different age groups, 2008 to 2018

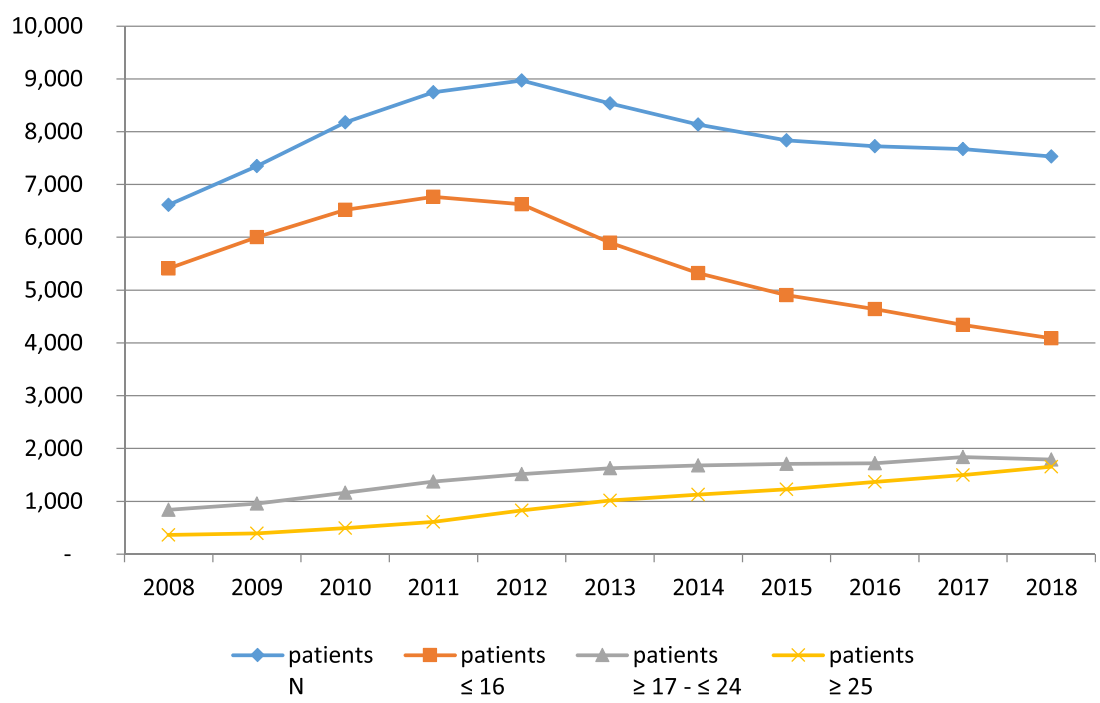


Fig. 2 Prescribed ADHD

medicines for patients with a diagnosis of ADHD, in DDDs; 2008 to 2018

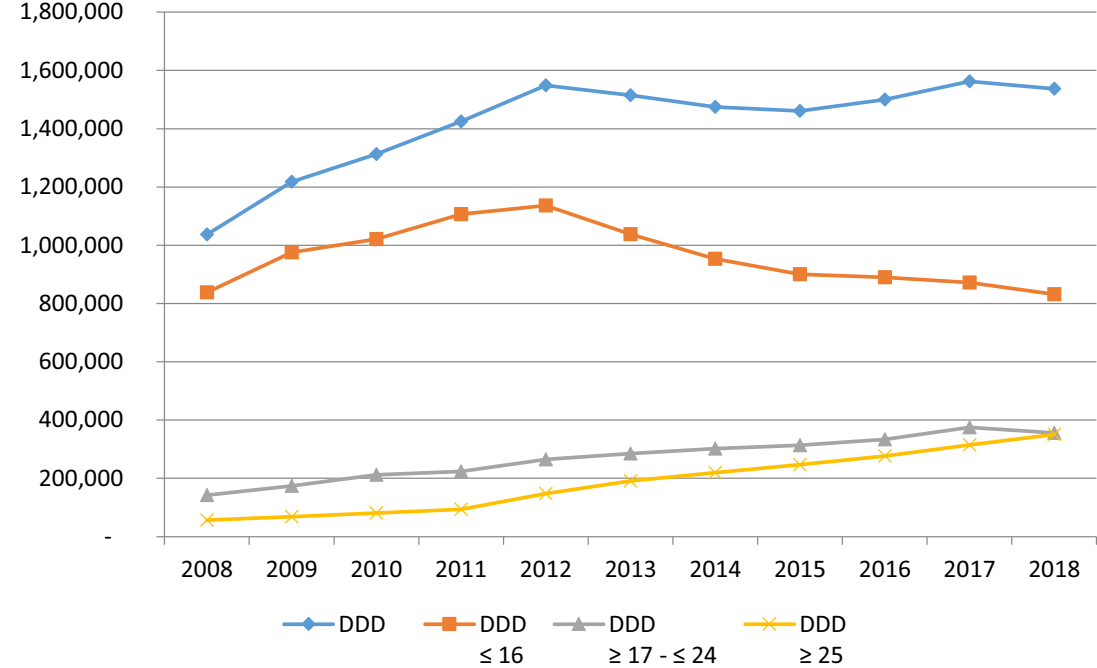

atomoxetine grew, but not steadily, in adults and decreased in children/adolescents.

When we considered the patient's age at therapy initiation, we observed some age differences (Fig. 3). During the study period, drug therapy was very frequently initiated mostly in younger persons ( $\leq 16$ years), with a peak at the age of 10 years, followed by a more or less linear decrease in the age of first prescription until the age of 21 . This finding corresponds to the age of the "last prescription", i.e., the point of time when no further prescription was issued. The group of children aged 13 showed the highest number of patients receiving a "last prescription", which then steadily decreased until the age of 25 .

\section{Persistence}

Figure 4 shows how long patients received a prescription for a medication, calculating the months between the first and last prescription. This analysis is based on those patients who received a prescription $(n=29,396)$. These patients received their prescriptions of an ADHD medicine for 22 months, on average (median: 12; first quartile: 34 ; third quartile: 2 ). The results differed substantially according to the age of the patients. While especially the patients younger than 12 years remained under treatment, on average, for 2 years or longer, adult patients received medication for a much shorter time (Fig. 3). A high percentage (27\%) of older adults, so-called spot users, received an ADHD medicine only once, compared with $17 \%$ of spot users among children.

The sensitivity analysis revealed similar trends, but inevitably, with a generally lower level of persistence in the case of the 180 d-criterion (see Figure; Appendix B).

\section{Comorbidities and drug prescriptions}

Approximately two-thirds $(52,319 / 77,504)$ of all patients had at least one other diagnosis from Chapter 5 ICD-10-CM (mental, behavioural and neurodevelopmental disorders; F 10-F 99). This figure was somewhat higher in children/ adolescents $(76 \%)$ than in those aged 17 to 24 years $(60 \%)$
Fig. 3 Number of patients, according to the patient's age at therapy initiation (first prescription) and last prescription

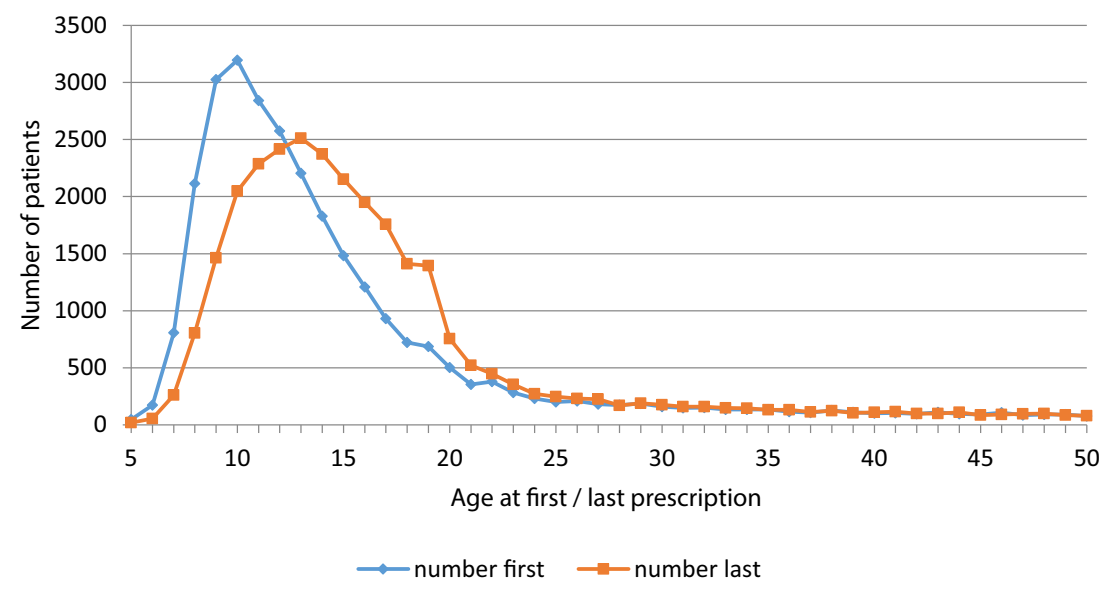


Fig. 4 Drug persistence, according to age at first prescription

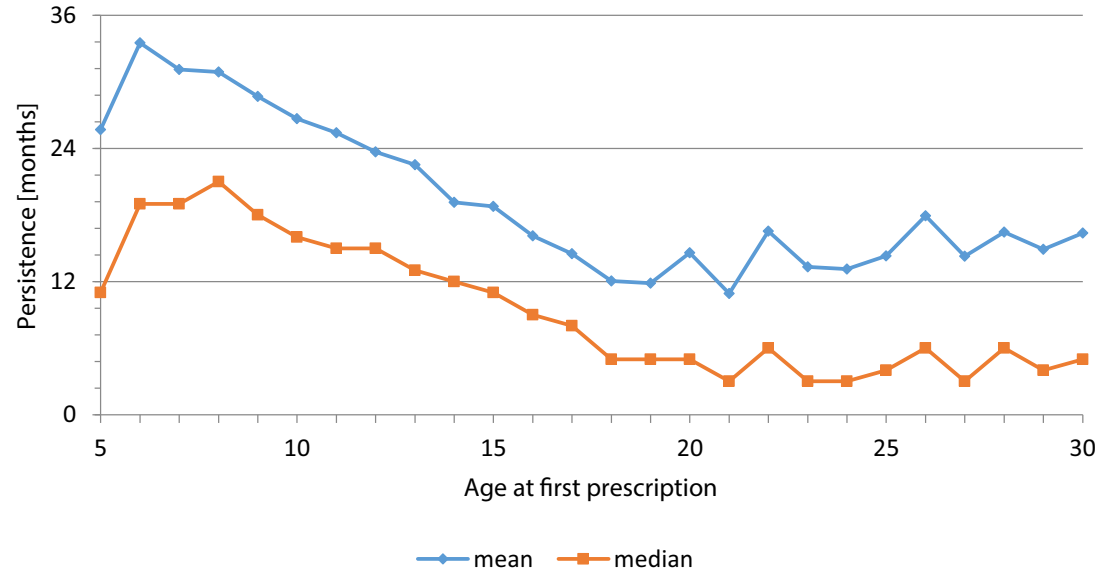

or $\geq 25$ years $(63 \%)$. More importantly, the single diagnoses differed markedly between the age groups (Table 1). Not surprisingly, the younger patients were more often diagnosed as having "pervasive and specific developmental disorders" (54\%), especially speech and language disorders, while these diagnoses were not common in adults. Vice versa, one-third of the adult patients were diagnosed with "mood (affective) disorders" (F30-F39), whereas nearly none of the children received this diagnosis. "Mental disorders" (F40-F48) were more frequent in adults, but "reaction to severe stress, and adjustment disorders" (F43) was identified in approximately $10 \%$ of the patients in all age groups.

Interestingly, these comorbidities played only a minor role in a doctor's decision regarding whether to prescribe an ADHD drug for patients with a diagnosis of ADHD. While $38.6 \%$ of the persons with only an ADHD (F90) diagnosis and no other diagnosis from ICD-Chapter 5 ICD-10-CM received a drug, it was $37.6 \%$ of those with at least one additional Fdiagnosis (Table 1). Even when a patient had more than 2, 3 or 4 psychiatric diagnoses, the likelihood of receiving an

Table 1 Psychosomatic/psychiatric comorbidity and ADHD drugs

\begin{tabular}{|c|c|c|c|c|c|c|c|c|c|}
\hline \multirow[b]{2}{*}{ Psychiatric/psychosomatic diagnoses } & \multicolumn{3}{|c|}{ Children $(\leq 16$ years $)$} & \multicolumn{3}{|c|}{$\begin{array}{l}\text { Adolescents, younger } \\
\text { adults (17-24 years) }\end{array}$} & \multicolumn{3}{|c|}{ Older adults ( $\geq 25$ years) } \\
\hline & $\mathrm{N}$ & $(\%)$ & $\begin{array}{l}\text { ADHD } \\
\text { drugs } \\
\%\end{array}$ & $\mathrm{~N}$ & $(\%)$ & $\begin{array}{l}\text { ADHD } \\
\text { drugs } \\
\%\end{array}$ & $\mathrm{~N}$ & $(\%)$ & $\begin{array}{l}\text { ADHD } \\
\text { drugs } \\
\%\end{array}$ \\
\hline Only F90 (ADHD) & 8071 & $(24.3)$ & 28.4 & 9887 & $(39.6)$ & 51.0 & 7216 & $(37.3)$ & 32.9 \\
\hline Additional F-diagnoses & 25,121 & $(75.7)$ & 31.3 & 15,042 & $(60.4)$ & 53.6 & 12,124 & $(62.7)$ & 31.0 \\
\hline All & 33,192 & $(100)$ & 30.6 & 24,929 & $(100)$ & 52.6 & 19,340 & $(100)$ & 31.7 \\
\hline \multicolumn{10}{|l|}{ Additional F-diagnoses } \\
\hline Mental disorders due to known physiological conditions (F01-F09) & 208 & $(0.6)$ & 31.3 & 175 & $(0.7)$ & 45.7 & 1624 & $(8.4)$ & 5.6 \\
\hline $\begin{array}{l}\text { Mental and behavioural disorders due to psychoactive } \\
\text { substance use (F10-F19) }\end{array}$ & 105 & $(0.3)$ & 30.5 & 670 & $(2.7)$ & 53.0 & 2479 & $(12.8)$ & 25.4 \\
\hline $\begin{array}{l}\text { Schizophrenia, schizotypal, delusional, and other non-mood psy- } \\
\text { chotic disorders (F20-F29) }\end{array}$ & 45 & $(0.1)$ & 48.9 & 189 & $(0.8)$ & 47.6 & 554 & $(2.9)$ & 37.7 \\
\hline Mood [affective] disorders (F30-F39) & 1064 & $(3.2)$ & 38.7 & 2202 & $(8.8)$ & 56.4 & 6308 & $(32.6)$ & 33.7 \\
\hline $\begin{array}{l}\text { Anxiety, dissociative, stress-related, somatoform and other } \\
\text { nonpsychotic mental disorders (F40-F48) }\end{array}$ & 5939 & $(17.9)$ & 33.0 & 4645 & $(18.6)$ & 51.6 & 6588 & $(34.1)$ & 24.1 \\
\hline $\begin{array}{l}\text { Behavioural syndromes associated with physiological disturbances } \\
\text { and physical factors (F50-F59) }\end{array}$ & 1162 & $(3.5)$ & 25.7 & 598 & $(2.4)$ & 50.5 & 911 & $(4.7)$ & 24.5 \\
\hline Disorders of adult personality and behaviour (F60-F69) & 2524 & (7.6) & 26.0 & 1779 & $(7.1)$ & 51.4 & 1706 & $(8.8)$ & 35.1 \\
\hline Intellectual disabilities (F70-F79) & 1497 & $(4.5)$ & 29.5 & 792 & $(3.2)$ & 46.8 & 276 & $(1.4)$ & 30.8 \\
\hline Pervasive and specific developmental disorders (F80-F89) & 17,778 & $(53.6)$ & 29.3 & 6854 & $(27.5)$ & 56.1 & 625 & $(3.2)$ & 56.0 \\
\hline $\begin{array}{l}\text { Behavioural and emotional disorders with onset usually occurring in } \\
\text { childhood and adolescence (F91-F98) }\end{array}$ & 14,252 & $(42.9)$ & 33.5 & 7248 & $(29.1)$ & 55.2 & 1467 & (7.6) & 61.2 \\
\hline Unspecified mental disorder (F99) & 359 & (1.1) & 19.8 & 202 & $(0.8)$ & 53.5 & 119 & $(0.6)$ & 26.9 \\
\hline
\end{tabular}


ADHD drug did not increase; quite the opposite, the rate decreased slightly from $40 \%$ of patients receiving a drug if they had one additional psychiatric diagnoses to $34 \%$ of those with four additional psychiatric diagnoses. While the rate of patients with a prescription was higher in adolescents, it made no difference whether the patients had an additional psychiatric diagnosis or not.

\section{Discussion}

\section{Summary of the main findings}

The overall prescription of medicines to treat ADHD in Germany remained stable over the last few years or increased only slightly after new guidelines worldwide recommended a more liberal use of these medicines. ADHD and its pharmacological management are increasingly observed among older adolescents and adults. Older adults ( $\geq 25$ years) use ADHD medicines, on average, for a much shorter period of time than children and adolescents. Psychiatric comorbidities were frequent among persons with an ADHD diagnosis but did not seem to be decisive for a drug prescription.

\section{Strengths and limitations of the study}

We had access to a large and continuous data set from community practices, covering general practices as well as paediatric, neurological and psychiatric practices in Germany. The data comprised the last 10 years, with diagnoses and drug prescriptions as well as the age and sex for persons with an ADHD diagnosis. The sample of practices was deemed to be representative of German practices [22] and was evenly distributed throughout the country.

A limitation of the data source is that it only documents prescribed medications but not whether they are really dispensed from a pharmacy. However, comparing our main results with global prescription data from German health insurance companies, our study approach seemed to yield valid results. Lohse and Müller-Oerlinghausen [24, 25], for example, reported a sharp increase in methylphenidate from 13 million DDDs in 2000 to 58 million DDDs in 2012, with a slight reduction to 53 million DDDs in 2018; however, this decrease was compensated by an increase in lisdexamfetamine from approximately 1 million DDDs when first released in 2013 to 8 or 10 million DDDs in the last 3 years. These findings are exactly in line with our results.

We could follow up the participants using a pseudonymized code but only within one practice and not across several practices. We probably underestimated, to a certain degree, drug supply and persistence of those who received their prescriptions from more than one practice or changed the practice during the time period under study. Patients, however, do not change practice frequently in Germany, with more than $85 \%$ of the patients visiting only the same, or maximum two general practitioners (GPs) and more than $90 \%$ visiting only the same or a maximum of 2 paediatricians, neurologists or psychiatrists [26]. However, we could not follow up patients when changing the practice and the same patients may consult several practices of the IQVIA practice panel. So, it was not possible to define the population at risk and we abstained from calculating the disease prevalence for the different age groups.

\section{Comparison with the literature and meaning of the results}

The frequency and quantity of prescriptions of methylphenidate are still a major concern in the scientific and public communities (not only) in Germany [27]. Portions of the public and some scientists fear that new ADHD guidelines will lead to a further increase in these drugs [16]. As far as children are concerned, our study did not detect any indications for such a trend to date. Prescriptions for children are still on a high level, compared with the levels two decades ago.

The cessation or slight decrease in methylphenidate use in recent years seen in our study was also observed in another German pharmacoepidemiological study [28]. According to the authors of this study, this trend could be caused by prescription restrictions by the German regulatory agency, a more cautious stance towards pharmacotherapy and medicalization among physicians and parents, and warnings regarding the potential cardiovascular risks of methylphenidate medications. However, this latter argument is only partially convincing as a counter-argument against the medicalization hypothesis since the authors did not consider compensation by other ADHD medicines in youth.

Moreover, and in remarkable contrast to these concerns, we witnessed a constantly repeated warning of an allegedly insufficient awareness of "adult ADHD", including an ongoing debate whether a significant proportion of adults remain undiagnosed and, consequently, untreated [7, 17-19]. The results and trends observed in our study can be condensed to three arguments that may inform this debate.

First, many studies have documented an increase in adults diagnosed with ADHD who are receiving respective medicines worldwide, with relative increases in medication use per year of 17 to $19 \%$ for adults until 2015 , compared with only 9 to $15 \%$ for non-adults [29]. Two US studies also reported that the proportion of adults treated with stimulants grew rapidly from 1999 to 2010 and again from 2010 to 2014 , in contrast to youths, who had a modest increase in stimulant use [30, 31]. Extending the study period until 2018, we could show that this trend is still active in Germany, but only for adults and older adolescents who now represent nearly half of the ADHD population who receive medication. Therefore, pharmacotherapy for adults has developed greatly over the last decade, to an extent that 
compensated for the slight decline in ADHD medicines in children. This development does not necessarily exclude the possibility that a considerable part of adults are still undertreated but we witness increased attention towards adults and increased willingness to prescribe them ADHD medicines.

Second, based on an estimated transition rate from childhood to adult ADHD of approximately 50\% [32], several authors proposed that a suboptimal transition from child to adult services or adult mental health services and a reluctance to consider $\mathrm{ADHD}$ as a disease of later years and later onset may lead to an undertreatment of adults with symptoms of an ADHD [33, 34]. However, there was no particular discontinuation of treatment during the transition from adolescence to early adulthood (Fig. 3). We could not detect a specific high number of patients receiving their "last prescription" at the age of 16,17 or 18 years; rather, the rate of "last prescriptions" continuously declined from the age of 13 years. While older age was a predictive factor for lower persistence in our study as in a Taiwan study [35], we could not detect a specific low persistence in the transition group so that it is rather unlikely that the transition phase is a trigger for the discontinuation of treatment.

Third, recent research [4] suggests that the emergence of ADHD symptoms in later years may not originate from ADHD itself but rather reflects one or more other mental health disorders with symptoms similar to ADHD as follows: depression is associated with difficulty focusing on tasks, anxiety may cause distractibility, substance use may lead to low arousal levels and lack of motivation, and so on. This concept may help to explain why so many adults $(27 \%)$ in our sample were spot users, i.e., they did not receive or demand a second prescription after an initial therapy with an ADHD medicine. Although we do not know who, the patient or the doctor, stopped pharmacotherapy after an initial trial of an ADHD drug for a rather short time, this lack of persistence may indicate that one party or both parties tried a sort of "empirical" therapy in the case of symptoms similar to ADHD symptoms - but often stopped them if not successful after a first drug trial. One could assume a higher rate of adults experiencing intolerable adverse effects, or incompatibility with their drugs for other medical problems, e.g., cardiac drugs. Indeed, a meta-analysis [36] found that adult ADHD patients randomized to CNS stimulant treatment demonstrated a statistically significant increased resting heart rate and systolic blood pressure findings compared with subjects randomized to placebo. However, none of the studies reported any serious cardiovascular events nor was the rate of drug discontinuation due to cardiovascular symptoms different between the treatment and placebo groups.

The obvious sensitivity towards such ADHD symptoms together with therapeutic attempts also contradicts the hypothesis that doctors neglect adult ADHD. Although some adults may still be undertreated or do not receive medicines at all, the lack of persistence observed in our study may be, at least partly, explained by "overtreatment" of those adults who were treated empirically with ADHD medicines for symptoms similar to ADHD symptoms but stopped the treatment probably because they did not experience a benefit. Different patterns of methylphenidate use among adults were also observed in Pauly et al.'s study where drug persistence decreased with age and adults who used methylphenidate had more psychiatric disorders than the younger ones [23].

\section{Conclusion}

The ever-increasing prescription of ADHD medicines, a major concern of the public and many researchers, stopped some years ago and even decreased in some age groups. While new guidelines obviously did not lead to an increasing trend in methylphenidate use among children, we should not overrate the decline in the use of these medicines. The reduction was very small, so the use of methylphenidate is still high.

However, there are still not enough data about the benefits and risks of long-term therapy, and post-marketing surveillance studies in community populations may be helpful. Even so, a stronger focus on the role of comedicationparticularly interacting comedication or medication classes like atypical antipsychotics possibly prescribed for ADHD may be helpful to better understand the decision to prescribe or not to prescribe an ADHD medicine and drug persistence.

What is surprising is the call to give adults and their pharmacological supply more attention. Considering the strong rise in drug supply of adults and the notable prescriptions pattern for this patient group, our analysis shows that doctors pay greater attention towards adults with a possible diagnosis of ADHD and a possible undertreatment of them.

Our pharmacoepidemiological analysis does not allow for inferences about the adequacy of the drug trends or the benefits of the medicines prescribed. At best, this analysis deepens our knowledge about recent trends and indicates risks and blind spots [2] in the scientific and public debate about the use of psychiatric medications for ADHD.

Acknowledgments Open Access funding provided by Projekt DEAL. We are indebted to IQVIA (Frankfurt, Germany) which permitted access to parts of their database. Professor Karel Kostev, Scientific Principal of IQVIA helped to make sense of the database whenever necessary. A contract guaranteed the authors complete independence in study design, in analysis and interpretation of data; in the writing of the report; and in the decision to submit the article for publication.

Authors' contributions Both authors conceived the study and performed the literature review. TG had the leading role in data analysis and interpretation; WH had the leading role in writing the first draft of the paper. Both authors corrected and approved the final version of the article and are the scientific guarantors for this study. 
Data availability statement The data that support the findings of this study are available from third party (data owners). Restrictions apply to the availability of these data, which were used under licence for this study. Data are available from the authors with the permission of third party.

\section{Compliance with ethical standards}

Original publication, authorship and conflict of interest The paper is not under consideration by another journal, and none of the results presented here have been published previously. Both authors fulfil the criteria for authorship and give their consent for publication. There was no conflict of interest.

Ethics Ethical approval was not necessary due to the nature of the data (secondary data analysis of anonymized data). The German law allows the use of anonymous electronic medical records for research purposes under certain conditions. According to this legislation, it is not necessary to obtain informed consent from patients or approval from a medical ethics committee for observational studies that contains no directly identifiable data. Therefore, the study is exempt from formal ethical approval and no waiver of ethical approval from an Institutional Review Board or ethics committee is required.

Open Access This article is licensed under a Creative Commons Attribution 4.0 International License, which permits use, sharing, adaptation, distribution and reproduction in any medium or format, as long as you give appropriate credit to the original author(s) and the source, provide a link to the Creative Commons licence, and indicate if changes were made. The images or other third party material in this article are included in the article's Creative Commons licence, unless indicated otherwise in a credit line to the material. If material is not included in the article's Creative Commons licence and your intended use is not permitted by statutory regulation or exceeds the permitted use, you will need to obtain permission directly from the copyright holder. To view a copy of this licence, visit http://creativecommons.org/licenses/by/4.0/.

\section{References}

1. Sharma A, Couture J (2014) A review of the pathophysiology, etiology, and treatment of attention-deficit hyperactivity disorder (ADHD). Ann Pharmacother 48(2):209-225. https://doi.org/10. 1177/1060028013510699

2. Franke B, Michelini G, Asherson P, Banaschewski T, Bilbow A, Buitelaar JK, Cormand B, Faraone SV, Ginsberg Y, Haavik J, Kuntsi J, Larsson H, Lesch KP, Ramos-Quiroga JA, Réthelyi JM, Ribases M, Reif A (2018) Live fast, die young? A review on the developmental trajectories of ADHD across the lifespan. Eur Neuropsychopharmacol 28(10):1059-1088. https://doi.org/10. 1016/j.euroneuro.2018.08.001

3. Caye A, Sibley MH, Swanson JM, Rohde LA (2017) Late-onset ADHD: understanding the evidence and building theoretical frameworks. Curr Psychiatry Rep 19(12):106. https://doi.org/10.1007/ s11920-017-0858-7

4. Asherson P, Agnew-Blais J (2019) Annual research review: does late-onset attention-deficit/hyperactivity disorder exist? J Child Psychol Psychiatry 60(4):333-352. https://doi.org/10.1111/jcpp. 13020

5. Thomas R, Sanders S, Doust J, Beller E, Glasziou P (2015) Prevalence of attention-deficit/hyperactivity disorder: a systematic review and meta-analysis. Pediatrics 135(4):e994-e1001. https:// doi.org/10.1542/peds.2014-3482
6. Kooij JJS, Bijlenga D, Salerno L, Jaeschke R, Bitter I, Balázs J, Thome J, Dom G, Kasper S, Nunes Filipe C, Stes S, Mohr P, Leppämäki S, Casas M, Bobes J, Mccarthy JM, Richarte V, Kjems Philipsen A, Pehlivanidis A, Niemela A, Styr B, Semerci B, Bolea-Alamanac B, Edvinsson D, Baeyens D, Wynchank D, Sobanski E, Philipsen A, McNicholas F, Caci H, Mihailescu I, Manor I, Dobrescu I, Saito T, Krause J, Fayyad J, RamosQuiroga JA, Foeken K, Rad F, Adamou M, Ohlmeier M, Fitzgerald M, Gill M, Lensing M, Motavalli Mukaddes N, Brudkiewicz P, Gustafsson P, Tani P, Oswald P, Carpentier PJ, de Rossi P, Delorme R, Markovska Simoska S, Pallanti S, Young $\mathrm{S}$, Bejerot S, Lehtonen T, Kustow J, Müller-Sedgwick U, Hirvikoski T, Pironti V, Ginsberg Y, Félegyházy Z, GarciaPortilla MP, Asherson P (2019) Updated European consensus statement on diagnosis and treatment of adult ADHD. Eur Psychiatry 56:14-34. https://doi.org/10.1016/j.eurpsy.2018.11.001

7. Bachmann CJ, Philipsen A, Hoffmann F (2017) ADHD in Germany: trends in diagnosis and pharmacotherapy. Dtsch Arztebl Int 114(9):141-148. https://doi.org/10.3238/arztebl.2017. 0141

8. Bachmann CJ, Wijlaars LP, Kalverdijk LJ, Burcu M, Glaeske G, Schuiling-Veninga CCM, Hoffmann F, Aagaard L, Zito JM (2017) Trends in ADHD medication use in children and adolescents in five western countries, 2005-2012. Eur Neuropsychopharmacol 27(5): 484-493. https://doi.org/10.1016/j.euroneuro.2017.03.002

9. Renoux C, Shin J-Y, Dell'Aniello S, Fergusson E, Suissa S (2016) Prescribing trends of attention-deficit hyperactivity disorder (ADHD) medications in UK primary care, 1995-2015. Br J Clin Pharmacol 82(3):858-868. https://doi.org/10.1111/bcp.13000

10. Ehrhardt C, Boucherie Q, Pauly V, Braunstein D, Ronflé E, Thirion X, Frauger E, Micallef J (2017) Methylphenidate: gender trends in adult and pediatric populations over a 7year period. Therapie 72(6): 635-641. https://doi.org/10.1016/j.therap.2017.05.005

11. Storebø OJ, Ramstad E, Krogh HB, Nilausen TD, Skoog M, Holmskov M, Rosendal S, Groth C, Magnusson FL, MoreiraMaia CR, Gillies D, Buch Rasmussen K, Gauci D, Zwi M, Kirubakaran R, Forsbøl B, Simonsen E, Gluud C, Cochrane Developmental, Psychosocial and Learning Problems Group (2015) Methylphenidate for children and adolescents with attention deficit hyperactivity disorder (ADHD). Cochrane Database Syst Rev 11:CD009885. https://doi.org/10.1002/14651858.CD009885. pub2

12. Graf WD, Miller G, Nagel SK (2014) Addressing the problem of ADHD medication as neuroenhancements. Expert Rev Neurother 14(5):569-581. https://doi.org/10.1586/14737175.2014.908707

13. National Institute for Health and Care Excellence (UK) (2018) Attention deficit hyperactivity disorder: diagnosis and management. National Institute for health and care Excellence: Clinical Guidelines, London

14. Deutsche Gesellschaft für Kinder- und Jugendpsychiatrie, Psychosomatik und Psychotherapie e.V. et al. (2017) S 3 Guideline ADHD in childhood, adolescence and adulthood [in German]; available at: https://www.awmf.org/uploads/tx szleitlinien/028-0451_S3_ADHS_2018-06.pdf

15. Berndt C (2018) Quick Ritalin pill for children with ADHD [in German]. Süddeutsche Zeitung, 14 June 2018; available at: https://www.sueddeutsche.de/gesundheit/medizin-schnelleritalinpille-fuer-den-zappelphilipp-1.4015592

16. Faltinsen E, Zwi M, Castells X, Gluud C, Simonsen E, Storebø OJ (2019) Updated 2018 NICE guideline on pharmacological treatments for people with ADHD: a critical look. BMJ Evid Based Med 24(3):99-102. https://doi.org/10.1136/bmjebm-2018-111110

17. Zalsman G, Shilton T (2016) Adult ADHD: a new disease? Int J Psychiatry Clin Pract 20(2):70-76. https://doi.org/10.3109/ 13651501.2016.1149197 
18. Schubert I, Lehmkuhl G (2017) The natural course and treatment of ADHD, and its place in adulthood. Dtsch Arztebl Int 114(9):139140. https://doi.org/10.3238/arztebl.2017.0139

19. Katzman MA, Bilkey TS, Chokka PR, Fallu A, Klassen LJ (2017) Adult ADHD and comorbid disorders: clinical implications of a dimensional approach. BMC Psychiatry 17(1):302. https://doi.org/ 10.1186/s12888-017-1463-3

20. Beau-Lejdstrom R, Zito JM (2018) Getting to long-term effectiveness and safety of attention-deficit hyperactivity disorder medications. Clin Pharmacol Ther 104(4):610-612. https://doi.org/10. 1002/cpt.1137

21. Rathmann W, Bongaerts B, Carius H-J, Kruppert S, Kostev K (2018) Basic characteristics and representativeness of the German disease analyzer database. Int J Clin Pharmacol Ther 56(10):459466. https://doi.org/10.5414/CP203320

22. Becher H, Kostev K, Schroder-Bernhardi D (2009) Validity and representativeness of the "disease analyzer" patient database for use in pharmacoepidemiological and pharmacoeconomic studies. Int J Clin Pharmacol Ther 47(10):617-626. https://doi.org/10. 5414/cpp47617

23. Pauly V, Frauger E, Lepelley M, Mallaret M, Boucherie Q, Micallef J (2018) Patterns and profiles of methylphenidate use both in children and adults. Br J Clin Pharmacol 84(6):1215-1227. https://doi. org/10.1111/bcp. 13544

24. Lohse MJ, Müller-Oerlinghausen B (2010) Psychotropic drugs [in German]. In: Schwabe U, Paffrath D (eds) Arzneiverordnungsreport 2010: Aktuelle Daten, Kosten, Trends und Kommentare, XIV. Springer-Verlag, Berlin Heidelberg, pp 797-842

25. Lohse MJ, Müller-Oerlinghausen B (2019) Psychotropic drugs [in German]. In: Schwabe U, Paffrath D, Ludwig W-D et al (eds) Arzneiverordnungs-Report 2019. Springer, Berlin Heidelberg, pp 927-959

26. Grobe TG, Steinmann S, Szecsenyi J (2019) BARMER Arztreport 2019: Schriftenreihe zur Gesundheitsanalyse, 1. Auflage edn. Asgard Verlagsservice, Siegburg

27. Conrad P, Bergey MR (2014) The impending globalization of ADHD: notes on the expansion and growth of a medicalized disorder. Soc Sci Med 122:31-43. https://doi.org/10.1016/j. socscimed.2014.10.019

28. Langner I, Haug U, Scholle O, Lindemann C, Schröder C, Riedel O (2019) Potential explanations for increasing methylphenidate use in children and adolescents with attention-deficit/hyperactivity disorder in Germany from 2004 to 2013. J Clin Psychopharmacol 39(1): 39-45. https://doi.org/10.1097/JCP.0000000000000980
29. Raman SR, Man KKC, Bahmanyar S, Berard A, Bilder S, Boukhris T, Bushnell G, Crystal S, Furu K, KaoYang YH, Karlstad Ø, Kieler H, Kubota K, Lai ECC, Martikainen JE, Maura G, Moore N, Montero D, Nakamura $\mathrm{H}$, Neumann $\mathrm{A}$, Pate V, Pottegård A, Pratt NL, Roughead EE, Macias Saint-Gerons D, Stürmer T, Su CC, Zoega H, Sturkenbroom MCJM, Chan EW, Coghill D, Ip P, Wong ICK (2018) Trends in attention-deficit hyperactivity disorder medication use: a retrospective observational study using population-based databases. Lancet Psychiatry 5(10):824-835. https://doi.org/10.1016/S2215-0366(18)30293-1

30. Oehrlein EM, Burcu M, Safer DJ, Zito JM (2016) National trends in ADHD diagnosis and treatment: comparison of youth and adult office-based visits. Psychiatr Serv 67(9):964-969

31. Burcu M, Zito JM, Metcalfe L, Underwood H, Safer DJ (2016) Trends in stimulant medication use in commercially insured youths and adults, 2010-2014. JAMA Psychiatry 73(9):992-993

32. Fayyad J, Sampson NA, Hwang I et al (2017) The descriptive epidemiology of DSM-IV adult ADHD in the World Health Organization world mental health surveys. Atten Defic Hyperact Disord 9(1):47-65. https://doi.org/10.1007/s12402-016-0208-3

33. Newlove-Delgado T, Hamilton W, Ford TJ, Stein K, Ukoumunne OC (2019) Prescribing for young people with attention deficit hyperactivity disorder in UK primary care: analysis of data from the clinical practice research Datalink. Atten Defic Hyperact Disord 11(3):255-262. https://doi.org/10.1007/s12402-019-00288-6

34. Eke H, Ford T, Newlove-Delgado T, Price A, Young S, Ani C, Sayal K, Lynn RM, Paul M, Janssens A (2019) Transition between child and adult services for young people with attention-deficit hyperactivity disorder (ADHD): findings from a British national surveillance study. Br J Psychiatry:1-7. https://doi.org/10.1192/ bjp.2019.131

35. Wang L-J, Yang K-C, Lee S-Y, Yang CJ, Huang TS, Lee TL, Yuan SS, Shyu YC (2016) Initiation and persistence of pharmacotherapy for youths with attention deficit hyperactivity disorder in Taiwan. PLoS One 11(8):e0161061. https://doi.org/10.1371/journal.pone. 0161061

36. Mick E, McManus DD, Goldberg RJ (2013) Meta-analysis of increased heart rate and blood pressure associated with CNS stimulant treatment of ADHD in adults. Eur Neuropsychopharmacol 23(6):534-541. https://doi.org/10.1016/j.euroneuro.2012.06.011

Publisher's note Springer Nature remains neutral with regard to jurisdictional claims in published maps and institutional affiliations. 